\title{
BÚSQUEDA E INCLUSIÓN LABORAL DE PERSONAS CON DISCAPACIDAD COGNITIVA EN CHILE: UNA MIRADA DESDE SUS MADRES.
}

\author{
JOB SEARCH AND INCLUSION COGNITIVE DISABLED PEOPLE IN CHILE: A \\ LOOK FROM THEIR MOTHERS.
}

\author{
$\mathrm{M}^{\mathrm{a}}$ Theresa von Furstenberg L.; Florencia Iriarte S; Daniela Navarro O. ${ }^{1}$
}

\begin{abstract}
RESUMEN
El presente artículo identifica los factores sociales que inciden en el proceso de búsqueda e inclusión laboral de jóvenes que presentan una discapacidad cognitiva en Chile, intentando dilucidar qué aspectos se constituyen en barreras para la incorporación a un trabajo desde la perspectiva de sus madres. Se basa en una investigación cualitativa en la que se utilizaron entrevistas en profundidad y cuestionarios semi estructurados, para identificar las visiones y significaciones de las madres de jóvenes con discapacidad cognitiva en torno al proceso de búsqueda e incorporación laboral. Como principales resultados, se han identificado tres tipos de obstáculos al empleo de los jóvenes con discapacidad cognitiva en el discurso de las entrevistadas: obstáculos sociales, personales y domésticofamiliares. En el plano social, las participantes señalan la falta de interés por parte de empresas privadas, la prevalencia de estereotipos y la creencia común que las personas con discapacidad son menos productivas. En el plano interno/nivel de familia, los padres se reconocen temerosos ante el proceso de integración, lo que asocian a que las personas con discapacidad cognitiva son más vulnerables y se encuentran expuestas a sufrir abusos o perjuicios, por lo que toman una postura selectiva ante las posibles ofertas de empleo. En lo que respecta al nivel personal, encontramos las declaraciones asociadas a una baja autoestima, inseguridad, falta de autonomía comunicativa y problemas de salud de las personas con discapacidad cognitiva, que influyen en la capacidad de búsqueda y en la adecuación personal al puesto de trabajo.
\end{abstract}

Palabras clave: Discapacidad cognitiva; Inclusión laboral; Barreras sociales.

\begin{abstract}
The aim of this paper is to stress out the the social factors that chilean young people with cognitive disability have to face in the process being inserted in a job. It intents to figure out, from the mothers' point of view, the aspects that constitutes a barrier for their children to be employed. This survey has been conducted from a multidisciplinary setting and a qualitative design, using interviews and a semi structured survey. As main outcomes, we have indentified three kinds of barriers: social, personal and domestic/family. At a social level, mothers point out the lack of interest from private corporations, the prevalence of stereotypes and the common belief that disabled people are less productive. At the domestic/family level, most parents are reluctant to agree with the labor integration process, mainly because they think that their children might be abused or deceived by the employers and their teamwork. Therefore, they are extremely cautious when selecting job opportunities. As a personal level, mothers found statements associated to low self-esteem, unsteadiness, lack of autonomy and communicative disabilities.
\end{abstract}

Keywords: Cognitive Disabilities, Including labor, social barriers.

\footnotetext{
${ }^{1} \mathrm{M}^{\mathrm{a}}$ Theresa von Furstenberg L. Licenciado en Psicología; Mg. en Recursos Humanos; (C) Doctor en Psicologia. Directora Diploma en Habilidades Laborales; Florencia Iriarte S; Psicopedagoga, Coordinadora Académica Diploma en Habilidades Laborales; Daniela Navarro O, Socióloga, Mg. en Estudios Latinoamericanos, Asesora Metodológica Diploma en Habilidades Laborales. Dir: Fernández Concha No 700, Las Condes, Santiago de Chile. F: 6618766 /2207499; correo electrónico: mvonfurstenberg@unab.cl
} 


\section{INTRODUCCIÓN}

Actualmente habitan en Chile alrededor de 1.119.867 personas con discapacidad, de las cuales 137.965 presentan una discapacidad cognitiva (MIDEPLAN, 2006). La integración de las personas con discapacidad ha sido un objetivo reconocido por diferentes instancias internacionales y nacionales, declarando políticamente derechos para estas personas tendientes a la igualdad y comprometiéndose a crear programas que se dirijan a erradicar la discriminación y a favorecer la inclusión laboral (ONU, 2006). Sin embargo, el desempleo en Latinoamérica alcanza a más del $80 \%$ de las personas con discapacidad. En el caso de Chile, recientemente se ha aprobado la Ley 20.422 que establece normas dirigidas a la igualdad de oportunidades y a la inclusión social de las personas con discapacidad, a la que le subyace un enfoque centrado en las barreras sociales. En dicha ley, se revela un cambio en la visión hacia las personas discapacitadas, pasando de un enfoque biomédico centrado en la deficiencia personal a un enfoque social enfocado en el desarrollo de oportunidades y en la eliminación de las diferentes barreras hacia las inclusión de personas con discapacidad. Así, la ley reconoce a la persona discapacitada como "aquella que teniendo una o más deficiencias físicas, mentales, sea por causa psíquica o intelectual, o sensoriales, de carácter temporal o permanente, al interactuar con diversas barreras presentes en el entorno, ve impedida o restringida su participación plena y efectiva en la sociedad, en igualdad de condiciones con las demás" (Ley 20.422, Diario Oficial de la República, 2010, Art.5). Dicha definición de la discapacidad es la que se ha adoptado en la presente investigación, a la par que por discapacidad cognitiva hemos entendido como aquella caracterizada por las limitaciones significativas en el funcionamiento intelectual y la conducta adaptativa tal como se ha manifestado en habilidades prácticas, sociales, y conceptuales. Esta discapacidad comienza antes de los 18 años (Luckasson, R., Borthwick-Duffy, S., Buntix, W.H.E., Coulter, D.L., Craig, E.M., Reeve, A.,y cols., 2002).

La materialización de la ley se encuentra en programas generados desde el Servicio Nacional de la Discapacidad (SENADIS) entre los que se pueden mencionar: Ayudas técnicas (financiamiento para productos del mercado que ayuden a mitigar o controlar deficiencias y que aporten a la participación de personas con discapacidad), un Concurso Nacional de Proyectos para la Inclusión Social de Personas con Discapacidad (financiamiento de proyectos en salud, educación, trabajo y accesibilidad) y el Programa de Intermediación Laboral para Personas con Discapacidad (que busca facilitar el acceso al mercado laboral de personas con discapacidad a través de la orientación, intermediación y, en algunos casos, colocación laboral). Así, se han creado acciones públicas dirigidas al acceso al empleo en la población con discapacidad, principalmente a través del financiamiento a personas naturales o jurídicas (SENADIS, sitio web, 2010).

A diferencia de lo que sucede en Chile, en otros países las empresas han desarrollado un sistema de cuotas reservadas para personas con discapacidad, como es el caso de Suecia, Dinamarca y Noruega (Hernández, R, Cerrillo, R. y Izuzquiza, D., 2009) . A nivel empresarial, la ley en Chile sugiere que las empresas contraten a personas discapacitadas de manera preferente siempre que tengan igualdad de mérito con otros/as candidatos/as al empleo, lo mismo que para la administración pública. Junto con esto, la ley establece el derecho de las personas con discapacidad a celebrar con las empresas contratos de aprendizaje sin límite de edad, así como obligaciones para el empleador en cuanto a ajustes necesarios y a servicios de apoyo con que debe contar para un adecuado desempeño del trabajador con discapacidad (Prat, 2011), pero no obliga a tener un porcentaje de personas discapacitadas contratadas. 
Más allá de las políticas, en Chile del total de personas en edad productiva que presentan una discapacidad un $70.8 \%$ se encuentra desempleado (Zondek, A., Zepeda, M., González, F. y Recabarren, E. 2006). Lo que vuelve a plantear la necesidad de reflexionar qué sucede entre los lineamientos de política que defienden la inclusión social de las personas discapacitadas y la situación real de estas personas en nuestro país. En torno a esto, Zondek et al. (2006) mencionan que en Chile, los programas existentes para incorporar laboralmente a personas discapacitadas son insuficientes para cubrir la demanda nacional, junto con esto critica el que se centren principalmente en formar y capacitar a estas personas para el empleo pero no se encarguen de la colocación y el seguimiento laboral de las mismas.

Bajo una perspectiva social de la discapacidad y considerando el sistema económico capitalista que rige el mercado laboral, Ferreira (2008) distingue que la discapacidad no puede ser entendida como una problema médico que afecta aisladamente a los individuos, sino que se trataría de un colectivo que en función de la existencia de estructuras inadaptadas a sus capacidades, se encontraría sentenciado a la marginación. En este sentido, Barton (1998) plantea que la primacía de la visión patológica de la discapacidad en la sociedad ha generado una influencia negativa en el tipo de experiencia que tienen las personas discapacitadas. En un mismo sentido, Ferreira (2010) agrega que bajo la economía capitalista, la medicina fue responsable de establecer las normas que legitimarían al cuerpo de los individuos (sano/enfermo), la que traspasando las fronteras de su campo habría influido en la estructura ideológica y económica de las sociedades, asociándose el éxito social a la posesión de un cuerpo sano, bueno y bello, lo que reduciría las posibilidades de éxito por parte de las personas con algún tipo de discapacidad. Así, las sociedades capitalistas habrían desfavorecido a las personas con discapacidad situándolas en una posición de mayor exclusión, en tanto la concepción sobre la producción al interior de las empresas se centra en contar con trabajadores/as considerados/as útiles para aumentar la ganancia de los/as empresarios/as.

Desde la perspectiva de Hernández et al. (2009), el proceso de búsqueda e inclusión laboral para jóvenes con discapacidad cognitiva, presenta características complejas, en la medida en que en éste inciden diversos elementos que sinérgicamente se relacionan con la adaptación al mundo laboral de las personas con discapacidad. El éxito del proceso de integración laboral estaría dado por la coordinación de diferentes elementos, a nivel social, familiar y de la propia persona con discapacidad.

Diversas investigaciones (Alba \& Moreno, 2004; Ated, 1998; Baldwin \& Johnson, 2001; Bardasi, Jenkins \& Rigg, 2000; Dávila, 2005; Livermore, 2000 en: Verdugo et. al, 2009), han centrado su análisis en la discriminación como una barrera determinante para el fracaso en la búsqueda e integración a un puesto laboral de las personas discapacitadas. En este sentido, Zastrow (2004, en Chima 2005) manifiesta que en general en la mayoría de las sociedades existe una falta de aceptación a las personas con discapacidad, basadas en impresiones erróneas hacia éstas.

Particularmente en el ámbito laboral, en cuanto a el/la empleador/a se distingue como discriminación el que éste/a considere que la integración de la persona discapacitada supone un mayor coste para la empresa o que se presente un miedo o temor a una menor productividad de dicha persona. Al respecto, diversos investigadores (Henderson, 1994; Schwartz, 1998; Maiden \& Younger, 1996, en Chima 2005) coinciden en que la creencia de que las personas con 
discapacidad tienen una menor capacidad de producción, se constituye en uno de los elementos claves que limita la consecución de un trabajo por parte de estas personas. En este sentido, algunos/as investigadores/as (Chima, 2005; Morrel, 1990 y Miel et al. 1993 en Arthur y Zarb, 1995) explican que tras el desempleo de las personas con discapacidad se ocultan ideas erróneas y estereotipadas en cuanto a las capacidades laborales de éstas. En este sentido, Morales y Sheafor (2004, en Chima 2005) distinguen que existen estereotipos de desamparo que ponen freno a las posibilidades de acción y a los aportes que pueden hacer dichas personas.

Se distingue también como discriminación el que no exista una concordancia entre los tipos de ofertas de empleo y los/as solicitantes del mismo, en este caso personas con discapacidad cognitiva. En ese sentido, sería discriminatorio el que las empresas no creen para las personas discapacitadas ofertas laborales adecuadas, es decir: jornadas laborales acotadas, rangos salariales adecuados y flexibilidad en los requisitos solicitados. Junto con esto, la búsqueda efectiva de empleo por parte de la persona con discapacidad, en muchos casos, es más costosa y se encuentra influida por problemas comunicacionales, lo que intervendría en el tipo de empleo al que la persona pueda acceder, así como también en el tiempo que esté dispuesta a utilizar en buscar un trabajo (Alba \& Moreno, 2004; Ated, 1998; Baldwin \& Johnson, 2001; Bardasi, Jenkins \& Rigg, 2000; Dávila, 2005; Livermore, 2000 en: Verdugo et. al, 2009). Al respecto, Berthoud (2007, en Purdam et al. 2008) menciona que las condiciones médicas que presenten las personas con discapacidad influirán en las tasas de empleo que presenten las mimas, mediando en el tiempo y los recursos con que éstas cuenten en el proceso de búsqueda laboral.

Por otro lado, Howard, Rogan y Jenaro (1998, en Hernández 2002), dan cuenta de elementos sociales y económicos, tales como la condición socio económica de la persona discapacitada y su lugar de residencia, así como la adecuación en cuanto a las infraestructuras.

Junto los factores mencionados anterioridad, se encuentran también aquellos que se asocian al ámbito familiar. La familia establece vínculos y relaciones con el medio social en general y marca las experiencias de la persona, además de satisfacer necesidades biológicas y determinar pautas educativas. Junto con esto, la familia da respuesta a necesidades de tipo afectivo, sexual, económico y social. Es el primer contexto socializador, el primer entorno en donde las personas que la constituyen crecen, evolucionan y desarrollan capacidades según los modelos vivenciados e interiorizados (Sarto, 2003). Rolland (2000), plantea que las familias enfrentan tres tipos de tareas: básicas (alimentación, vestuario, etc.); de desarrollo (de acuerdo a la etapa en que se encuentren) y azarosas (imprevistos, accidentes, discapacidad). Así, la familia tiene un rol importante para todas las personas, pero aún más importante para las personas que presentan una discapacidad. Siguiendo esto último, Rolland (2000) plantea que, idealmente, toda familia que se enfrenta a una enfermedad debiera contar con apoyo psicosocial que incluya a todos los miembros de la familia, desde un comienzo, que dé asesoría y normalice las posibles discusiones y problemas que puedan surgir. Contar con este apoyo ayudaría a disminuir el sentimiento de impotencia y de vergüenza que muchas veces vivencian las familias al contar entre sus integrantes con una persona discapacitada.

En cuanto a la cohesión familiar, Rolland (2000) refiere que en casos de discapacidad se suele hacer necesaria una mayor cohesión familiar, lo que suele traer problemas en el desarrollo normativo de sus miembros. Se hace necesario lograr un equilibrio entre apego y despego en los padres con el objetivo de no obstaculizar el desarrollo individual de cada integrante. Junto con 
esto, el autor destaca la relevancia de que las familias de personas con discapacidad tengan fronteras permeables, además de ser cohesionadas para enfrentar de manera positivas las dificultades que conlleva la discapacidad.

Un sistema cerrado se aislará y es posible que se generen disfunciones, junto con el empobrecimiento de las relaciones con pares. Es muy común que un niño con discapacidad o enfermo crónico quede aislado de sus pares y de las actividades de la comunidad (Rolland 2000).

Asimismo, los sistemas de creencias de las familias acerca de la enfermedad, determinarán las pautas de comportamiento frente a ella. En base a ellos construirán un significado para esa enfermedad y se organizarán. Kleinman (1988 en Rolland 2000) se refiere a que las familias construyen narrativas para las afecciones físicas que tienen 3 niveles: el biológico (descripción biomédica), la dimensión humana individual y familiar (la forma en que la persona enferma y su familia o red social mayor perciben los síntomas, la discapacidad y le dan respuesta) y la dimensión social de la enfermedad (significados que han quedado asociados a un problema físico debido a fuerza mayor, como por ejemplo intoxicación con plomo). Los tres niveles están en constante interacción y dan cuenta de un intento por parte de la familia, de poner orden en medio del caos que se les produce cuando deben hacer frente a la enfermedad (Rolland, 2000).

Por otra parte, el aumento de la esperanza de vida de las personas con discapacidad, trae consigo diversos y complejos desafíos para las familias, como lograr el equilibrio o el paso de proteger al niño a favorecer la autonomía a medida que crece; la integración del niño a su grupo de pares y a actividades acorde a la edad; dejar el cuidado total del niño para que sea él quien comience a cuidarse muchas veces genera en los padres un sentimiento de pérdida, ya que han estado habituados a ello. También se genera la necesidad de reafirmar constantemente a los niños, realizar lo necesario para que tengan una buena autoestima y una visión positiva de futuro y además, la familia debe aprender a relacionarse con el medio en que se encuentra, que muchas veces puede no estar preparado para atender las necesidades de estos niños y jóvenes, como colegios, sistemas legales y trabajo adecuado (Roberts y Wallander 1992, en Rolland 2000).

Los jóvenes con discapacidad en la etapa adolescente inevitablemente tendrán un conflicto con el tema de la autonomía. Tendrán la necesidad de ser como los demás, de ser parte de un grupo de pares y de elaborar proyectos acerca de su futuro. Por otra parte, la transición a la edad adulta temprana es un desafío para todos los miembros de la familia. Surgen temas como los límites, las expectativas poco realistas y la dependencia permanente, en el contexto de las tareas del desarrollo de la etapa, como la emancipación de los controles parentales, dejar el hogar, ser independiente, formar un nuevo hogar (Levinson, 1978 en Rolland 2000).

Desde la perspectiva de Valls, Vilà y Pallisera (2004) la familia tendría un rol primordial en el éxito en la búsqueda y mantención de un trabajo por parte la persona con discapacidad, señalando que ésta debe cooperar, interesarse y brindar apoyo en el proceso de búsqueda e incorporación laboral, además de construir un canal de cooperación con la organización o institución en la que se encuentre trabajando la persona que presenta la discapacidad.

Según Hernández et al. (2009), uno de los principales elementos con que deben contar las personas con discapacidad para poder optar a la inclusión laboral, es contar con una formación apropiada y especializada de acuerdo al puesto de trabajo, lo que garantizaría la permanencia en el mismo. Así también, sería necesario que ésta reciba un seguimiento constante en su desempeño laboral. Al respecto, Verdugo et al. (2009) mencionan que el que las personas con discapacidad 
presenten un nivel menor de formación, hayan adquirido en un nivel más bajo que el resto de las personas las habilidades laborales necesarias para desempeñarse en un empleo o bien cuenten con menos experiencia laboral, es reconocido como una discriminación laboral a dichas personas.

Por otra parte, se distinguen factores intra personales que influenciarían en las opciones que tengan las personas con discapacidad para integrarse a un puesto laboral. Según Chima (2005) una variable importante para la contratación de estas personas sería autoimagen. En este sentido, la presencia de una discapacidad tiende a incidir en que las personas discapacitadas se sientan inseguras frente al proceso de inclusión al mundo laboral, presentando temor a cometer errores y asumir riesgos, la sensación de ser inadecuado para el trabajo y una preocupación constante por la visión que los demás tengan hacia ellos/as (Gordon, Lam, \& Winter 1997, en Chima 2005). Lo anterior, produciría sensaciones de ansiedad e inseguridad en las personas con discapacidad y una predisposición a que las cosas resulten de manera negativa, así como también influiría en la reacción que tengan las personas sin discapacidad hacia éstas (Chima, 2005).

\section{METODO}

Este artículo se ha basado en una investigación cualitativa sobre los factores que inciden en el éxito o fracaso en la integración laboral de personas con discapacidad cognitiva. En ese marco, se llevó a cabo un cuestionario de caracterización de la situación laboral de jóvenes con discapacidad aplicado a padres/madres de jóvenes egresados/as de un diploma universitario en habilidades laborales para personas con discapacidad cognitiva, mediante el cual se identificaron los principales elementos a abordar en la realización de entrevistas semi estructuradas mediante las que se profundizó en el tema de estudio. Aún cuando se invitó a participar de la investigación tanto a padres como a madres de personas con discapacidad, cabe mencionar que fueron las madres las que efectivamente quisieron o pudieron participar de la misma, debido a eso la investigación se centró en la perspectiva de las madres.

En total, participaron en la investigación 64 personas. En una primera etapa se aplicó el cuestionario de caracterización general del proceso de búsqueda e inclusión laboral de jóvenes con discapacidad cognitiva a 62 madres y 2 padres de los/as mismos/as. Posteriormente, se profundizó en el tema de estudio mediante 7 entrevistas semi estructuradas a madres de personas con discapacidad cognitiva. Cabe mencionar, que al ser los/as participantes padres/madres de jóvenes con discapacidad cognitiva que han cursado un diploma universitario de habilidades laborales además de analizar las barreras sociales para la integración laboral de éstos/as, se buscó indagar en la importancia del diploma como facilitador de dicha integración.

El presente artículo se centra en los resultados cualitativos de la investigación, en la medida en que lo que se pretende es preponderar las dimensiones simbólicas del proceso de integración al mundo laboral de personas con discapacidad cognitiva. Se utilizó la técnica de entrevista semi estructurada en tanto ésta permite identificar creencias, actitudes, valores, opiniones y conocimientos de el/la entrevistado/a sobre la temática de estudio. Así, a continuación se presentan los principales resultados obtenidos a través del análisis de contenido de las entrevistas realizadas, identificando los puntos de consenso y desacuerdo entre las participantes. 


\section{RESULTADOS}

Al realizar un análisis del discurso de las entrevistadas se puede observar una visión pesimista en cuanto al nivel de inclusión laboral que han alcanzado las personas con discapacidad cognitiva en Chile, lo que se fundamenta en que observan que muchas de estas personas no han logrado acceder a un trabajo y quienes lo han hecho acceden a una actividad laboral de carácter precario. Sin embargo, no se observa en las entrevistadas una actitud completamente crítica ante las oportunidades laborales a las que pueden acceder sus hijos/as, así manifiestan intentar ser positivas, a la vez que hacen mención a elementos espirituales o religiosos en los que se apoyan para hacer frente a las diversas dificultades que conlleva el tener un hijo/a con discapacidad cognitiva.

Las participantes consideran que la sociedad chilena, presenta deficiencias para poder incorporar laboralmente a personas que tienen una discapacidad cognitiva. Dichas deficiencias van desde la forma en que se estructura la sociedad (normas, recursos, etc.) a las actitudes (valores y creencias) que presentan sus miembros.

Las entrevistadas concordaron en que en general, en Chile existe discriminación y segregación hacia distintos grupos, entre los que se encuentran los/as discapacitados/as y, específicamente, aquellos/as que presentan una discapacidad cognitiva. Las razones atribuidas a dicha discriminación y segregación hacen referencia a una falta de comprensión por parte del resto de la sociedad de lo que son e implican las discapacidades cognitivas, tanto para quienes las presentan como para sus familias. Junto con esto, mencionan que en Chile las personas se comportan muchas veces de manera individualista y poco solidaria, especialmente con aquellos/as que presentan alguna discapacidad, en tanto son diferentes a lo considerado normal.

En este contexto, y sumando otros elementos que serán desarrollados posteriormente, mencionan que cobran relevancia los contactos familiares para que puedan incorporarse a un empleo, en la medida en que no existiría una predisposición inicial a la integración social de personas con discapacidad. Por el contrario, las entrevistadas creen que existen muchas veces actitudes de dureza y frialdad, mencionando en algunos casos experiencias de discriminación hacia sus hijos/as, burlas y abusos tanto a nivel tanto escolar como laboral. Lo que asocian a que los/as padres/madres de personas con discapacidad cognitiva consideren que el ambiente laboral es un espacio en el que sus hijos/as se encuentran más expuestas a sufrir riesgos o abusos que las personas sin discapacidad. A su vez, la mayoría de las entrevistadas menciona que recurrentemente la población en Chile manifiesta una actitud de desamparo ante las personas discapacitadas, significándolos/as como "pobrecitos/as", lo que consideran negativo para el desarrollo de su autoestima y su autoimagen.

En relación a lo anterior, le otorgan un valor importante al rol de las personas adultas que se encuentran a cargo de personas con discapacidad en la construcción y la apertura de espacios tanto laborales como en otros ámbitos (educativos, recreativos, etc.). En este sentido, las madres entrevistadas se significan a sí mismas como mediadoras entre el contexto social y sus hijos/as con discapacidad cognitiva, considerando que siempre tendrá que existir alguna persona que los/as acompañe y apoye en todo lo que vayan a emprender en el futuro. Así, mencionan recurrentemente que les produce preocupación lo que sucederá con éstos/as cuando ellas ya no estén, tanto a nivel de recursos económicos (principalmente aquellas que manifestaron tener 
menos recursos) como a nivel de relaciones sociales y de desarrollo personal. Lo último es aludido como una razón para considerar relevante el que sus hijos/as puedan integrarse al espacio laboral, en tanto creen que eso les aportaría a ellos/as una cierta autonomía e independencia así como la posibilidad de socializar, aunque no creen que sea factible que logren auto sustentarse a través de su propio empleo.

Siguiendo lo último, las madres entrevistadas cuyos/as hijos/as se encuentran desempeñando una actividad laboral $^{2}$ coinciden en que el trabajo al cual pueden optar personas con discapacidad cognitiva se tratará, en la mayoría de los casos, de un trabajo otorgado por compasión, favor o buena voluntad de personas que ocupan un cargo relevante al interior de una empresa o a través de una iniciativa familiar. Mencionan así, que el acceso a la actividad laboral por parte de sus hijos/as se llevó a cabo a través de contactos familiares o mediante el apoyo de la propia familia en el desempeño de una actividad comercial independiente. Agregan que las personas con discapacidad cognitiva que se encuentran trabajando lo hacen mayormente sin contrato, de manera voluntaria o independiente, en muchos casos sin un sueldo, con un sueldo pagado por las personas adultas que se encuentran a cargo (padre, madre, tío, abuela, etc.) o con una remuneración que con suerte llega al mínimo, según lo describen las participantes. Lo último lo relacionan con las bajas expectativas que tienen en cuanto a la permanencia de sus hijos/as en la actividad laboral que se encuentran desempeñando, aunque valoran positivamente el que éstos/as estén trabajando.

Aunque no de manera beligerante, todas las entrevistadas concuerdan en que existen pocas oportunidades laborales para jóvenes con discapacidad cognitiva, lo que relacionan a una baja voluntad empresarial para incorporarlos/as en un puesto de trabajo, en tanto estas personas no son consideradas productivas para las empresas. Algunas de las madres participantes expresan que es necesario que exista una mayor responsabilidad social empresarial ante las personas con discapacidad, reconociéndolas como sujetos y que la integración de éstas sea un deber y no una obra de bondad.

Junto con lo anterior, las participantes también coinciden en que existe una falta de capacitación en las empresas para emplear a estas personas. Mencionan que las empresas deberían contar con un/a tutor/a capacitado/a para guiar a la persona con discapacidad en el ejercicio de su actividad laboral, así como también que dichas tareas tienen que ser adecuadas a las capacidades de cada persona. Más allá del ámbito empresarial, las entrevistadas identifican la falta de capacitación en diferentes campos de la sociedad. Por ejemplo, en cuanto al sistema educativo mencionan que los colegios en Chile no se encuentran completamente capacitados para integrar a personas con necesidades educativas especiales, rememorando experiencias personales de dificultades para que sus hijos/as pudiesen cursar la enseñanza básica y media en un establecimiento ordinario. Junto con esto, a nivel universitario consideran que son escasas las iniciativas dirigidas a formar a personas que requieren una educación especial, sugiriendo que es necesario que se amplíen las oportunidades de formación técnica y profesional para estas personas, con el objetivo de facilitar su integración laboral.

En cuanto a los medios disponibles para que las personas con discapacidad cognitiva puedan buscar un empleo, las participantes concordaron en que tanto Internet como los Periódicos son un

\footnotetext{
${ }^{2}$ Fueron las propias participantes las que definieron la situación laboral de sus hijos/as (trabajando/desempleado).
} 
mecanismo inadecuado para que encuentren un trabajo apropiado, en la medida en que consideran que a través de estos medios no es posible saber si la empresa se encuentra capacitada para integrar laboralmente a éstos/as jóvenes ni expresar las dificultades que pueda tener el/la mismo/a, esto lo asocian a sus propios temores o aprehensiones lo que será expuestos más adelante.

En referencia a las políticas públicas dirigidas a las personas con discapacidad en Chile, las madres de jóvenes con discapacidad cognitiva entrevistadas mencionaron la necesidad de ampliar la cobertura de las acciones públicas y diversificarlas, a la par que hacen referencia a los altos costos económicos que conlleva convivir con una persona con discapacidad. Sin embargo, manifiestan a su vez no conocer con precisión la oferta pública chilena en la materia. Algunas de las entrevistadas expresaron que inscribir a su hijo/a en el Registro Nacional de Personas con Discapacidad sería contribuir a una estigmatización del/la joven como discapacitado/a y diferente a los/as demás. En ese sentido, todas las entrevistadas mencionaron no haber contado con apoyo público en el proceso de búsqueda e incorporación a un trabajo por parte de su hijo/a con discapacidad.

En general, las entrevistadas coinciden en que para que exista una integración, en todos los ámbitos de la sociedad es necesario que se genere y difunda más información tanto en los medios de comunicación como a nivel de investigaciones en referencia a las personas con discapacidad, considerando sus propias capacidades, demandas, necesidades y expectativas.

Para todas las participantes la integración laboral de sus hijos/as con discapacidad cognitiva es algo fundamental, coincidiendo en que en ese proceso el rol de la familia es prioritario. Así, mencionan que sin el apoyo familiar la búsqueda e inclusión a un empleo de estas personas sería imposible. Manifestando que aquellos/as que no cuenten con el apoyo familiar no lograrán encontrar un empleo y, en muchos casos, disminuirían considerablemente sus relaciones sociales.

En ese sentido, el proceso de búsqueda e incorporación al empleo se describe como un proceso que cuenta con la participación activa de los padres y, en algunos casos, de otros integrantes de la familia que, a través de personas conocidas o de la asistencia directa a las empresas u organizaciones, solicitan la incorporación de su hijo/a a un "empleo". Las comillas son porque recalcan que no se trataría de un empleo o trabajo ordinario (con contrato o free lance) sino que más bien sería el desempeño de alguna actividad laboral en algunos casos de manera voluntaria, tal como se mencionó anteriormente. En otros casos, las madres mencionan que algunos/as de éstos/as jóvenes que cursaron el diploma universitario gracias a éste lograron integrarse laboralmente, ya que realizaron una práctica profesional y posteriormente se quedaron trabajando. Sin embargo, mencionan que esos casos son reducidos en el marco de todos/as los/as jóvenes que presentan una discapacidad cognitiva hayan o no cursado un diploma universitario.

En ese proceso de búsqueda de empleo, en el que las madres entrevistadas se reconocen como intermediarias, consideran que un aspecto que influye y que puede constituirse en una barrera, es el sentimiento de vergüenza que en muchos casos los/as padres/madres de personas con discapacidad cognitiva experimentan al momento de tener que pedirle a personas conocidas o desconocidas que acepten a sus hijos/as para desempeñar una actividad laboral. Lo último, las entrevistadas lo asocian a que los/as padres/madres de personas con discapacidad cognitiva sienten que al acercarse a una empresa u organización para solicitar un trabajo para su hijo/a con 
discapacidad están pidiendo un favor, lo que implicaría reconocer la necesidad de ayuda por parte de la empresa u organización lo que significan como incómodo ya que piensan que las personas con discapacidad cognitiva, en la mayoría de los casos, no son consideradas productivas por parte de las empresas y organizaciones. Siguiendo lo último, las entrevistadas coinciden en que es más fácil para los/as padres/madres de jóvenes con discapacidad cognitiva pedirle empleo para sus hijos/as a alguien conocido, en tanto ya no sería necesario explicar las capacidades y necesidades que éstos/as presentan. Además, creen necesario para superar esa vergüenza que los/as padres/madres valoren el trabajo de sus hijos/as, afirmando que estas personas son un aporte para la sociedad en otros aspectos más allá de la productividad.

Junto con esto, las entrevistadas cuyos hijos/as se encuentran desempleados/as reconocen la falta de tiempo como una barrera para acompañar la búsqueda de empleo de éstos/as y lograr que se incorporen a un trabajo, sobre todo en el caso de madres que trabajan.

Por otro lado, entre los elementos asociados a la familia que inciden en el proceso de integración laboral distinguidos por las entrevistadas, se encuentra el contar con contactos familiares que tengan la posibilidad de decidir o incidir sobre la inclusión laboral de sus hijos/as con discapacidad cognitiva al interior de una empresa u organización. En ese sentido, las participantes mencionan que existen diferencias significativas en las posibilidades de integración laboral de éstos jóvenes, dependiendo de si la familia cuenta o no con contactos familiares que faciliten ese proceso, lo que variaría según el nivel socio económico de la familia.

Otro elemento importante en cuanto al apoyo de los/as padres/madres en la búsqueda de empleo, según las entrevistadas es que éstos/as motiven a sus hijos/as a realizar una actividad laboral, así como también les transmitan el valor que tiene el trabajo, en tanto creen que muchas veces los/as jóvenes con discapacidad cognitiva no dimensionan completamente la relevancia de trabajar y muestran poca motivación por buscar o conseguir un empleo.

Por otra parte, se observa una diferencia en el discurso entre las madres cuyos/as hijos/as se encuentran desempeñando alguna actividad laboral y las madres cuyos/as hijos/as están desempleados, en tanto las segundas expresan que los/as padres/madres de personas con discapacidad cognitiva muchas veces no asumen con facilidad dicha discapacidad, considerando necesario que exista una asimilación y aceptación de las necesidades educativas y laborales especiales que presenta la persona con discapacidad para una integración social y laboral adecuada.

A nivel emocional, todas las entrevistadas concuerdan en que el proceso de integración laboral de sus hijos/as con discapacidad cognitiva se encuentra cruzado por miedos en torno a diferentes aspectos que para ellas implica la incorporación al mundo del trabajo. En ese sentido, manifiestan temores asociados a actividades que sus hijos/as realicen fuera del hogar, los que se acentúan en el proceso de integración laboral, en tanto este supondría una mayor independencia de los/as jóvenes, pasando mayor tiempo fuera del domicilio familiar. Por ejemplo, se menciona el miedo a que les roben o sufran algún daño en el traslado al lugar de trabajo, expresando que desde ellas existe una cierta sobre protección hacia el/la hijo/a con discapacidad. En este sentido, expresaron que era importante que el lugar de trabajo quedara cerca del lugar de residencia de la familia, evitando traslados largos o complejos. Así, las entrevistadas coincidieron en que no tenían disposición a que sus hijos/as con discapacidad se trasladaran y trabajaran en otras comunas consideradas más peligras por tratarse de comunas de un nivel socioeconómico más bajo. 
Además, todas las entrevistadas coincidieron en que debido a las características personales de sus hijos/as (mayor timidez e inocencia que los/as demás, fundamentalmente) sienten miedo de que al incorporarse a un trabajo puedan ser inducidos a hacer cosas indebidas o desagradables, junto con esto mencionan sentir temor de que sean maltratados/as o ignorados/as por compañeros/as de trabajo u otras personas con las que tengan que interactuar. Aunque no exclusivamente, pero sí principalmente las madres de mujeres con discapacidad cognitiva entrevistadas manifestaron sentir temor a que sus hijas puedan sufrir un abuso sexual al interior del espacio de trabajo, por lo que consideran que es necesario que el lugar de trabajo sea seguro, lo último lo significan como un trabajo en un espacio abierto, de preferencia en el que trabajen mujeres, con actividades y responsabilidades adecuadas a las capacidades del/la joven con discapacidad.

Otro elemento que las entrevistadas distinguen como un temor, es el miedo a que sus hijos/as con discapacidad desempeñen mal su trabajo y tengan que enfrentarse a situaciones incómodas y frustrantes, en torno a esto algunas consideraron que transmitían ese temor a sus hijos/as, generando más inseguridad en los/as mismos/as. En ese sentido, las madres entrevistadas expresaron que para ellas era muy importante que el trabajo fuese el adecuado, declarando haber rechazado ofertas laborales que se le presentaron a la persona con discapacidad en tanto consideraban que éstas era inapropiadas (lugares riesgosos, con personas desconocidas, etc.).

En torno a lo anterior, las participantes de la investigación concordaron en que su posición como madres ante el proceso de integración laboral de sus hijos/as con discapacidad cognitiva es una posición selectiva ante las posibles opciones de trabajo a las que puedan optar éstos/as, en la medida en que manifestaron no observar una preparación de los espacios de trabajo (ni a nivel de infraestructura ni a nivel de recursos humanos) para integrar laboralmente y de una manera adecuada a éstos/as jóvenes.

Por otra parte, en cuanto a los elementos asociados a la persona con discapacidad cognitiva, las madres entrevistadas distinguen como una barrera el que éstos/as no cuenten con un nivel de formación especializada y suficiente en comparación a las personas sin discapacidad. En ese sentido, valoran positivamente la existencia del diploma universitario que cursaron sus hijos/as, sin embargo, consideran que las oportunidades de formación de este tipo son muy escasas si tomamos en cuenta al universo de personas con discapacidad en Chile, por lo que consideran pertinente ampliar los espacios de formación al interior de las universidades, a la vez que extender la duración de los cursos, con el objetivo de equiparar a estas personas con el resto de la población.

Otra barrera que observan las entrevistadas y en torno a la que también concuerdan, es el que desde su perspectiva muchas personas con discapacidad cognitiva presentan una baja autoestima y una falta de seguridad en sí mismos/as, lo que es considerado por éstas como una limitante para integrarse laboralmente en tanto afectaría en las comunicaciones que establezca la persona con discapacidad cognitiva, así como en las acciones que emprenda en el espacio de trabajo. En este sentido, las madres manifestaron que era necesario fomentar en estos/as jóvenes la autonomía, fortalecer su autoestima y la seguridad en sí mismos, siendo la inclusión laboral un elemento favorable para el logro de esos objetivos, a la vez que una meta para la cual se requiere contar con dichos requisitos (independencia, autoestima y seguridad en sí mismos/as). 
Junto con lo anterior, las participantes coinciden en que un elemento clave que influye en una menor contratación a personas con discapacidad cognitiva es que éstas pueden presentar una menor productividad que el resto de personas que componen la sociedad, lo que incide en que sean subvalorados/as como trabajadores/as en la medida en que no sería beneficioso desde la perspectiva empresarial. En cuanto a esto, las participantes expresaron que es necesario cambiar la visión hacia las personas con discapacidad cognitiva, en la medida en que éstas pueden trabajar y ser una aporte para las empresas, más allá del nivel de productividad que presenten, es decir, en otros aspectos como: las relaciones laborales, el sentido del trabajo, el valor de la vida, etc.

Por otro lado, otro elemento asociado a la persona con discapacidad y considerado una barrera por las entrevistadas es que en muchos casos consideran que los/as jóvenes con discapacidad cognitiva están acostumbrados a ser asistidos o apoyados por otras personas en diferentes ámbitos de la vida, lo que desde la perspectiva de las madres influye en que éstos/as no dimensionen realmente la relevancia de trabajar, lo que implicaría una mayor pasividad ante la búsqueda de trabajo.

Otro elemento personal que puede influir en la búsqueda y mantención en un empleo por parte de los/as jóvenes con discapacidad son los problemas de lenguaje y comunicación que puede presentar el/la joven, influyendo en las oportunidades laborales que tenga, así como en su desempeño para poder integrarse en un trabajo. En ese sentido, entre las madres de jóvenes desempleados se puede observar una mayor referencia a problemas de lenguaje y comunicación como limitantes para la inclusión laboral de sus hijos/as, que entre las madres cuyos/as hijos/as se encuentran trabajando.

\section{DISCUSIÓN}

A modo de conclusión, se puede afirmar que las entrevistadas expresaron más puntos de consenso que de desencuentro en sus discursos al referirse al proceso de inclusión laboral de sus hijos/as con discapacidad cognitiva. Coincidiendo en que éste se encuentra limitado por diferentes elementos a nivel social, familiar y personal que se interrelacionan y que conllevan a que el desempeño de alguna actividad laboral por parte de estos/as jóvenes sea mucho menor en comparación al resto de la población chilena en edad productiva.

Considerando los planteamientos de Henderson (1994, en Chima, 2005) Schwartz, (1998, en Chima, 2005) y Maiden \& Younger (1996, en Chima, 2005) y analizando el discurso de las entrevistadas cabe reflexionar en torno a la existencia de discriminación en diferentes espacios de la sociedad chilena (colegio, universidades, trabajo, espacios recreativos, etc.) hacia las personas con discapacidad cognitiva. Así, las entrevistadas reconocen la presencia de prejuicios negativos hacia la situación y las capacidades de dichas personas (desamparados/as, improductivos/as, etc.), basados en una falta de información, que influirían en una baja voluntad tanto empresarial como política de generar oportunidades laborales y formativas adecuadas para estas personas.

En este sentido, se observa que las madres distinguen la presencia de discriminación en el mercado de trabajo, concordando en que en Chile existe una ausencia de medios adecuados de búsqueda de empleo para las personas con discapacidad cognitiva, una falta de ofertas de empleo adecuadas a las capacidades de esas personas (requisitos exigidos y condiciones de empleo inadecuadas), una falta de cobertura y diversificación de los programas públicos dirigidos a la 
inclusión laboral (tal como lo defiende Zondek et al., 2006), una falta de iniciativas de formación y especialización laboral para esas personas, así como también una baja contratación efectiva incluso para cargos que podrían desempeñar sin mayor dificultad. En definitiva, desde la perspectiva de las entrevistadas existe una baja preparación del "mundo laboral" y una falta de voluntad (sustentada en valores negativos asociados a la discapacidad así como en general a los grupos que presentan actitudes o condiciones diferentes al resto de la sociedad) para incluir efectivamente a un trabajo a las personas con discapacidad cognitiva, ambos factores son distinguidos por Chima (2005) como una muestra de la discriminación existente hacia dichas personas.

En función de lo anterior, los trabajos a los cuales acceden las personas con discapacidad, desde lo descrito por las entrevistadas, son trabajos precarios, a menudo sin contrato o sin un vínculo laboral formal (tampoco free lance), en muchos casos no remunerados o con una remuneración simbólica o mínima, en otros casos los sueldos son pagados por los/as propios padres/madres de la persona que presenta la discapacidad. En base al discurso de las participantes, las personas con discapacidad cognitiva efectúan más que un trabajo una actividad laboral, que logran realizar gracias a que alguna persona conocida o de buena voluntad esté dispuesta a incorporarlos/as a su empresa u organización. Así, las madres entrevistadas distinguen que los contactos con los que cuente la familia de la persona con discapacidad serán necesarios, aunque no suficientes en muchos casos, para que dicha persona pueda desempeñar una actividad laboral. Esto último es asociado con la posición económica de la familia, en tanto la mayoría de las entrevistadas mencionan que a mayor nivel socioeconómico la familia contará con una mayor cantidad de contactos y, por ende, de alternativas laborales para sus hijos/as con discapacidad, así como con recursos económicos para hacer frente a los altos costes que conlleva la discapacidad, lo que concuerda con los planteamientos de Howard, Rogan y Jenaro (1998, en Hernández 2002).

Tomando los planteamientos teóricos de la investigación y el análisis del discursos de las entrevistadas, es pertinente plantearse en qué medida las personas con discapacidad logran encajar en el espacio laboral, en el marco de la empresa, en tanto en dicho espacio existirían prejuicios negativos, una valoración simbólica de las personas con discapacidad cognitiva como personas menos productivas y más costosas que las personas sin discapacidad. Lo último pone en jaque el paradigma o los valores que sustentan el mercado laboral, la contratación de las personas, los requisitos, los procesos de selección, etc. en el sistema capitalista de mercado y hace necesario revisar en qué medida se lograrán eliminar las barreras sociales construidas en torno a la discapacidad, aún cuando se declare como un objetivo a nivel político, si a nivel cultural y en la estructura de las instituciones, organizaciones y empresas primaría, desde lo expuesto por las entrevistadas y considerando los planteamientos teóricos de la investigación, una visión excluyente o subvalorada de la diferencia, particularmente cuando se asocia a una baja productividad. En este sentido, no bastaría con una declaración política de principios inclusivos hacia las personas con discapacidad sino que sería necesario que se produjeran transformaciones a un nivel, en principio, cultural que se sustenten en una revaloración de estas personas, ya no como "pobrecitos o improductivos" si no como personas que pueden realmente aportar valores positivos a través del desempeño de una actividad laboral, para lo que sería necesario que se genere y difunda más información sobre la discapacidad cognitiva en sus diferentes tipos.

Junto con lo último, sería necesario introducir cambios a nivel organizacional (recursos humanos, valores de la organización, normas, leyes, etc.) e infraestructural en las instituciones, 
organizaciones y empresas, haciendo que éstas se adecuen a las necesidades de las personas con discapacidad cognitiva. Considerando ambos aspectos (transformación en los valores asociados a la discapacidad y cambios a nivel de prácticas, organización y capacidades), se podría avanzar hacia que las personas que presentan una discapacidad cognitiva y sus familias no tengan que reducir sus espacios de búsqueda e inclusión a un empleo a las oportunidades que puedan ofrecer los contactos familiares y su correspondiente "buena voluntad" o "compasión", en tanto distinguirían medios de búsqueda adecuados, ofertas apropiadas y capacidades idóneas para la inclusión laboral de personas con discapacidad cognitiva, así como una voluntad real de inclusión, lo que podría influir positivamente en una disminución de los temores presentes en los/as padres/madres hacia este proceso, así como en aportar a una autoimagen positiva en estos/as jóvenes, ambos elementos fundamentales para el éxito en la búsqueda e inclusión laboral, según el discurso de las participantes en esta investigación.

\section{REFERENCIAS BIBLIOGRAFICAS}

- Arthur, Sue y Zarb, Gerry. (1995) Measuring Disablement in Society: Working Paper 4 Barriers to Employment for Disabled People. Disponible en: http://www.leeds.ac.uk/disability-studies/archiveuk/Zarb/barriers\%20to\%20employment.pdf

- Barton, L (Comp.). (1998). Discapacidad y sociedad. (2da ed), Madrid, España: Ediciones Morata.

- Chima, Felix O. (2005). Personas con discapacidad y el empleo: Implicaciones para el Trabajo Social y los Roles y Apoyo de la Rehabilitación. Diario de Trabajo Social en la Discapacidad y Rehabilitación, 4 (3), p. $39-60$.

- Hernández, J.L. (2002) Análisis y propuestas de integración al mercado laboral de personas con discapacidad. Revista Telemática de Filosofía del Derecho, (6), 71-95.

- Hernández, R.; Cerrillo, R. y Izuzquiza, D. (2009). La inclusión de Discapacitados Intelectuales en el Mundo Laboral: Análisis Cualitativo. Revista Latinoamericana de educación inclusiva. Disponible en: http://www.rinace.net/rlei/numeros/vol3num2/art2.pdf, visitada el 13 de Septiembre del 2011.

- Ley 20.422, Diario Oficial de la República, Gobierno de Chile, 2010, Art.5.

- Luckasson, R., Borthwick-Duffy, S., Buntix, W.H.E., Coulter, D.L., Craig, E.M., Reeve, A.,y cols. (2002). Mental Retardation. Definition, classification and systems of supports (10th ed.).

- Washington, DC: American Association on Mental Retardation. [Traducción al castellano de M.A. Verdugo y C. Jenaro (en prensa). Madrid: Alianza Editorial].

- Ministerio de Planificación (MIDEPLAN) (2006). Gobierno de Chile Encuesta CASEN 2006: Discapacidad. Recuperado el 15 de Septiembre del 2011 de: http://sibuc.uc.cl/sibuc/dhi/citar/normas_apa_electronicos.html 
- ONU (2006). Convención sobre los derechos de las personas con discapacidad. Disponible en: http://www.un.org/spanish/disabilities/convention/qanda.html, visitada el 14 de Septiembre del 2011.

- Prat, I. (2011) Inclusión Laboral de personas con discapacidad. Santiago, Chile: Servicio Nacional de la Discapacidad - Gobierno de Chile.

- Purdam, K., Afkhami R., Olsen W. y Thornton P. (2008) Discapacidad en el Reino Unido: midiendo igualdad. Revista Disability \& Society, 23, (1), 53-65.

- Rolland, John. (2000). Familias, enfermedad y discapacidad. España: Gedisa.

- Sarto, M.; (2003). Familia y Educación. En Gonzáles, F.; Calvo, M.; Verdugo, M. (Eds.) (2003). Familia y Educación (pp. 39-52). Salamanca: Publicaciones del INICO.

- Servicio Nacional de la Discapacidad (SENADIS). (2010). Manual sobre la Ley $N^{o}$ 20.422: Establece Normas sobre Igualdad de Oportunidades e Inclusión Social de Personas con Discapacidad. Recuperado el 18 de Noviembre del 2011 de: http://www.senadis.gob.cl/descargas/centro/legislacion nacional/Manual Ley20422.pdf

- Servicio Nacional de la Discapacidad (página web). Programas y fondos concursables. Recuperado el 14 de Noviembre del 2011, de: http://www.senadis.gob.cl/

- Seelman, C. (2004) Tendencias en la rehabilitación y la discapacidad: transición desde un Modelo médico a un Modelo de integración. Disability World, (22). Disponible en: http://www.disabilityworld.org/01-03_04/spanish/acceso/rehabtrends.shtml

Valls, M.; Vilà, M. y Pallisera, M. (2004) La inserción de las personas con discapacidad en el trabajo ordinario. El papel de la familia. Revista de Educación, (334), 99-117.

- Verdugo, M., Jordán de Urríes, F., Sánchez, M. y Benito, M. (2009) Influencia de la protección social y el sistema de pensiones en la actividad y el acceso al empleo de personas con discapacidad. Salamanca, España: Universidad de Salamanca - Ministerio del trabajo e inmigración: Gobierno de España.

- Zondek, A. (2006). Abriendo oportunidades. Santiago, Chile: Ed. Gobierno de Chile. 51 págs.

- Zondek, A., Zepeda, M., González, F. y Recabarren, E. (2006). Discapacidad en Chile: Pasos hacia un Modelo integral del funcionamiento humano. Santiago, Chile: Fondo Nacional de Discapacidad. 